\title{
Stability of up-milling and down-milling, part 2: experimental verification
}

\author{
B.P. Mann ${ }^{\text {a }}$, T. Insperger ${ }^{\text {b }}$, P.V. Bayly ${ }^{c}$, G. Stépán ${ }^{\text {d,* }}$ \\ a Department of Mechanical Engineering, Washington University, 1 Brookings Drive, St. Louis, MO 63130, USA \\ ${ }^{\mathrm{b}}$ Department of Applied Mechanics, Budapest University of Technology and Economics, Budapest, H-1521, Hungary \\ c Department of Mechanical Engineering, Washington University, 1 Brookings Drive, St. Louis, MO 63130, USA \\ d Department of Applied Mechanics, Budapest University of Technology and Economics, Budapest, H-1521, Hungary
}

Received 25 June 2002; accepted 20 August 2002

\begin{abstract}
The stability of interrupted cutting in a single degree of freedom milling process was studied experimentally. An instrumented flexure was used to provide a flexible workpiece with a natural frequency comparable to the tooth pass frequency, mimicking high speed milling dynamics. The displacement of the system was sampled continuously and periodically once per cutter revolution. These data samples were used to asses the stability of the system. Results confirm the theoretical predictions obtained in Part 1. (C) 2002 Elsevier Science Ltd. All rights reserved.
\end{abstract}

Keywords: High-speed milling; Regenerative effect; Tooth pass excitation

\section{Introduction}

Predictive models for machining operations provide an opportunity to improve process efficiencies and dimensional precision. Dynamic machining models give manufacturers the capability to predict regions of stable and unstable cutting for a large combination of process parameters. This allows analytical methods and/or simulation to be used in place of costly trial and error for process optimisation. The advances in digital computers over the last decade have made the latest research efforts more accessible for industrial applications. This is true for industrial application from conventional turning [1], to advanced high-speed milling [2].

The implementation of dynamic machining models requires accurate identification of model parameters such as cutting force coefficients and dynamic parameters for the machine tool structure. Several alternatives are available for estimation of mechanistic cutting coefficients [3]; here the methods of Halley [4] are applied. Dynamic

\footnotetext{
* Corresponding author. Tel.: +36-1-463-1369; fax: +36-1-4633471.

E-mail address: stepan@mm.bme.hu (G. Stépán).
}

parameters can also be identified by the methods of Inman [5] for adequately spaced modes or by the methods of Juang [6] for closely spaced modes.

Unstable machining vibrations can be detected by the analysis of the chatter signal [7-10]. Trajectory reconstruction methods of stochastic processes can be effectively used for identifying the relative motion of the tool and the workpiece from noisy time series [11]. The stability charts published in the specialist literature are commonly accompanied by frequency diagrams illustrating chatter frequencies at the loss of stability $[12,13]$. The reason for this practice is that these frequencies can be identified experimentally and this is a direct way to theoretical models and predictions.

In this second part of the research report, the theoretical methods presented in the first part [14] are experimentally verified. The governing mathematical model developed in the companion paper is the delay-differential equation

$$
\begin{gathered}
\ddot{\xi}_{(t)}+2 \zeta \omega_{n} \dot{\xi}(t)+\omega_{n}^{2} \xi(t) \\
=-\frac{b K_{s}(t)}{m}[\xi(t)-\xi(t-\tau)],
\end{gathered}
$$

where $\xi(t)$ is the deviation between the ideal periodic 
motion and the real tool motion, $\zeta$ is the damping ratio, $\omega_{n}$ is the natural angular frequency, $K_{s}(t)$ is the $\tau$-periodic specific cutting force variation, $b$ is the nominal depth of cut, $m$ is the modal mass and $\tau=60 / N \Omega$ [s] is the tooth pass period, $\Omega$ is the spindle speed given in rpm and $N$ is the number of teeth.

\section{Stability prediction summary}

Both the finite element analysis in time (FEAT) and the semi-discretization (SD) method described in the first part of this study [14] approximate the milling system behaviour by discretizing the independent time variable of Eq. (1). The result is the formation of a discrete linear map equation in the form

$\mathbf{a}_{n}=\mathbf{Q} \mathbf{a}_{n-1}$.

The stability of the system is determined directly from the eigenvalues $\mu$ of the transition matrix $\mathbf{Q}$. These eigenvalues are also called characteristic exponents. For a range of operating conditions (depth of cut $b$ and spindle speed $\Omega$ ) the transition matrix is formed and the eigenvalues are found. If the magnitude of any eigenvalue exceeds one, the solution is unstable.

The predicted boundaries between stable and unstable cutting as a function of spindle speed and depth of cut are shown in Fig. 1. Two distinct types of instability are illustrated by eigenvalue trajectories in the complex plane: (1) a flip bifurcation or period doubling phenomenon occurs when the negative real eigenvalue passes through -1 ; (2) a Hopf bifurcation occurs when a complex eigenvalue obtains a magnitude greater than 1 . These eigenvalue trajectories are shown in the bottom graphs of Fig. 1 with the corresponding speed and depth of cut points shown in the top stability chart.
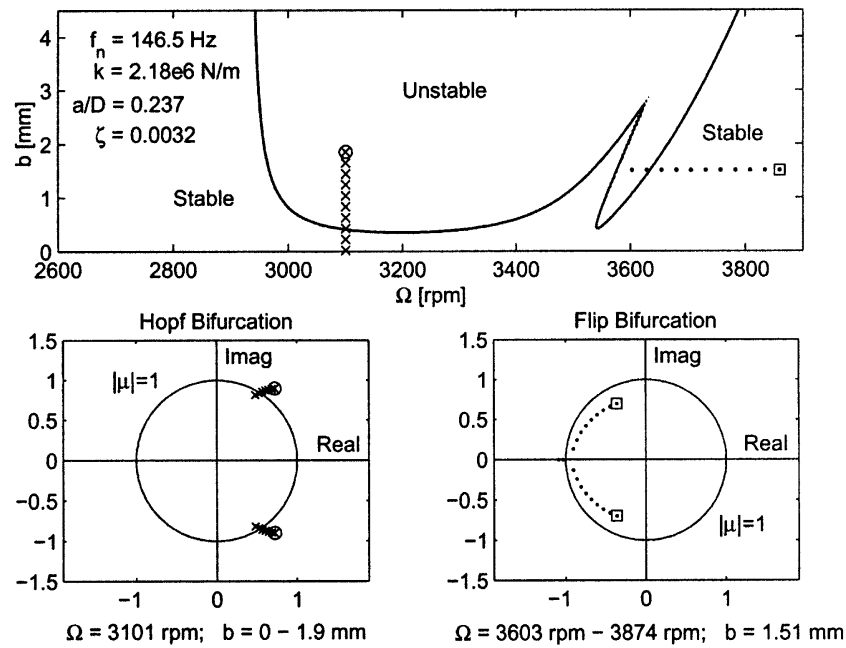

Fig. 1. Stability predictions and eigenvalue trajectories for up-milling.

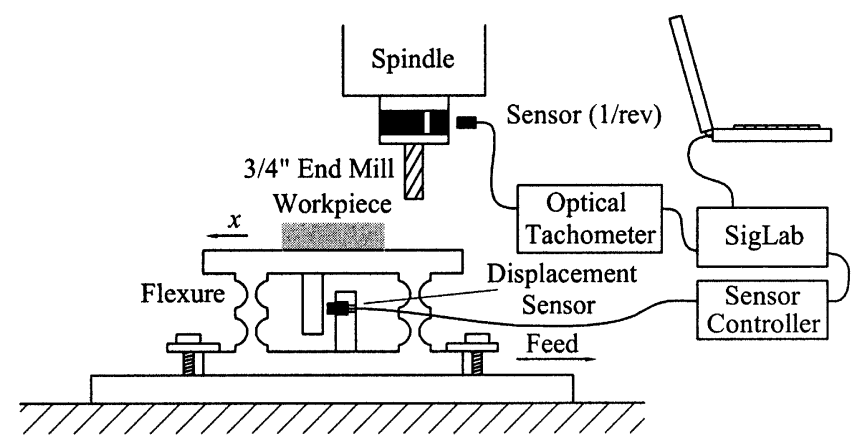

Fig. 2. Experiment schematic diagram.

\section{Experiment description}

Milling tests were performed with an experimental flexure designed to mimic the single degree of freedom (SDOF) system assumed in the first part of the paper [14]. A monolithic, unidirectional flexure was machined from aluminium and instrumented with a single non-contact, eddy current displacement transducer. A schematic diagram of the experiment is shown in Fig. 2.

\subsection{Workpiece dynamics}

Since the system under consideration contains a single dominant structural mode, several methods may be used for extraction of modal parameters [5]. One alternative is to compare the experimental transfer function in the $\mathrm{x}$-direction (shown in Fig. 3) to the single degree of freedom transfer function

$H(\omega)=\frac{1 / k}{1-\left(\omega / \omega_{n}\right)^{2}+i 2 \zeta \omega / \omega_{n}}$

of Eq. (1), where $\omega$ is forcing frequency, $k=m \omega_{n}^{2}$ is the system is the system stiffness. The natural frequency was
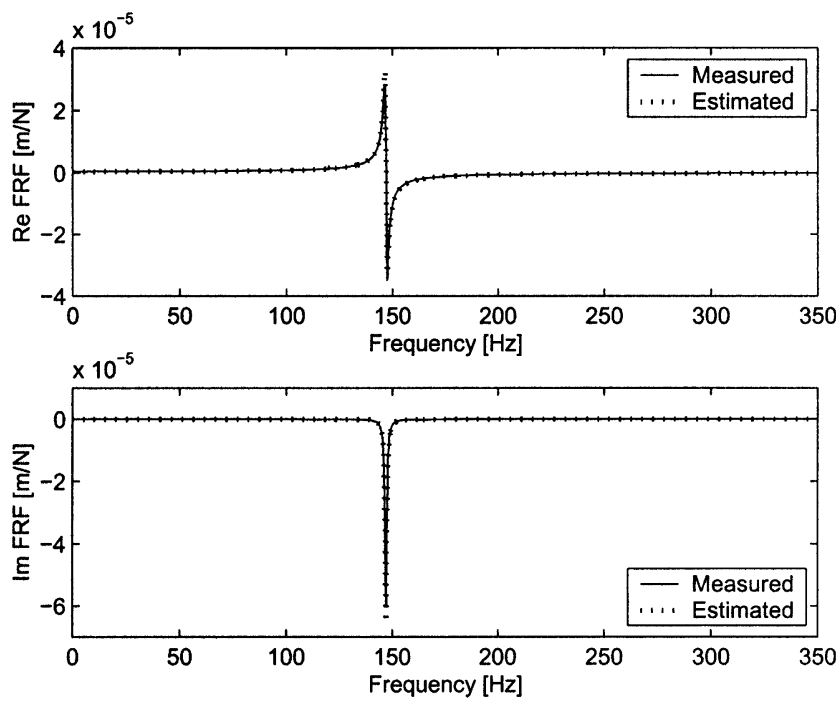

Fig. 3. Flexure transfer function. 
experimentally determined to be $f_{n}=\omega_{n} / 2 \pi=$ $146.5 \mathrm{~Hz}$ (consequently, $\omega_{n}=20.5 \mathrm{rad} / \mathrm{s}$ ).

The magnitude of Eq. (3) at $\omega=0$ gives the inverse of the system stiffness. Stiffness estimates were obtained by averaging the inverse value of the real portion of the transfer function over the first $20 \mathrm{~Hz}$. The stiffness of the flexure to deflections in the $x$-direction was measured to be $k=2.18 \times 10^{6} \mathrm{~N} / \mathrm{m}$. In comparison, the values of stiffness in the perpendicular $y$ - and $z$-directions were more than 20 times greater than in the $x$-direction. The damping ratio is given by substitution $\omega=\omega_{n}$ into Eq. (3):

$\zeta=\frac{1 / k}{2 \operatorname{Im}\left(H(\omega)_{\omega=\omega_{n}}\right)}$

According to Eq. (4), the damping ratio was estimated to be $\zeta=0.0032$ from the experimental transfer function imaginary peak. The system modal mass was computed to be $m=k / \omega_{n}^{2}=2.573 \mathrm{~kg}$.

\subsection{Cutting forces}

The cutting coefficients in the tangential and normal directions were determined from the rate of increase of cutting force as a function of chip load during separate cutting tests on a Kistler Model 9255B rigid dynamometer [4]. The estimated values were $K_{n}=$ $2.0 \times 10^{8} \mathrm{~N} / \mathrm{m}^{2}$ and $K_{t}=5.5 \times 10^{8} \mathrm{~N} / \mathrm{m}^{2}$. Measured and predicted cutting forces are shown in Fig. 4.

\subsection{Test procedures}

A radial immersion $a=4.515 \mathrm{~mm}$ was used to upmill and down-mill aluminium (7075-T6) test samples of width $6.35 \mathrm{~mm}(1 / 4 \mathrm{inch})$ over a specified range of spindle speeds and axial depths of cut. A $19.05 \mathrm{~mm} \mathrm{(3/4}$ inch) diameter carbide end mill with a single flute was used (the second flute was ground off to remove any effects due to asymmetry or runout). Consequently, the radial immersion ratio was $a / D=0.237$. Since the num-

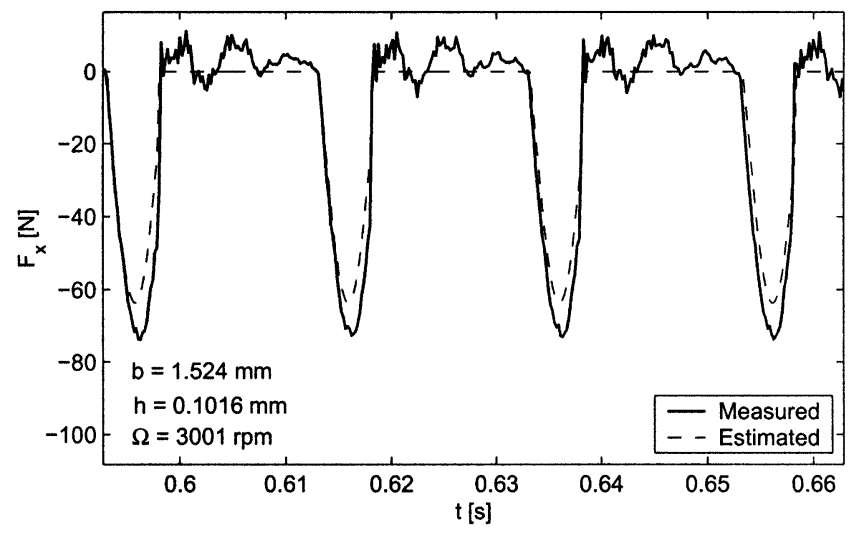

Fig. 4. Cutting force in the $x$-direction for half immersion up-milling. ber $N$ of teeth is one, the tooth pass period $\tau$ is equal to the rotation period $60 / \Omega$ of the tool. Feed was held at a constant $0.1016 \mathrm{~mm} / \mathrm{rev}(0.004 \mathrm{in} / \mathrm{rev})$. For these technological parameters, the theoretical specific cutting force variation $K_{s}(t)$ is shown in Fig. 5.

The displacement transducer output was anti-alias filtered and sampled (16-bit precision, 12800 samples/s) with SigLab 20-22a data acquisition hardware connected to a Toshiba Tecra 520 laptop computer. A periodic $1 /$ rev pulse was obtained with the use of a laser tachometer to sense a black-white transition on the rotating tool holder. The displacement data was recorded for approximately 15 seconds. The calibration of the displacement transducer was $1.2668 \times 10^{-4} \mathrm{~m} / \mathrm{V}$. The spectral analysis was performed using a Hanning window with no averaging procedures.

The sequential order for the cutting tests was to cut first in the regions of predicted stability. The next step was to perform cutting tests in the less robust stable regions between the stability lobes. The final series of cutting tests were all performed in the regions of predicted instability. The order of the cutting tests was adopted to prevent premature damage to both the tool and/or flexure.

\section{Experimental results}

Experimental results for up-milling and for downmilling have been superimposed onto a plot of the theoretical stability predictions in Figs. 6 and 7, respectively. Since stability boundaries for both the FEAT and the SD methods converge to the same result, as shown in the first part of this paper [14], a single stability boundary is shown. The boundaries in Figs. 6 and 7 were determined by the FEAT method with approximation parameter $E=4$, that is, the infinite dimensional DDE (1) was approximated by $10 \times 10$ dimensional discrete system. Cutting tests were declared stable if the $1 / \mathrm{rev}$ sampled position of the tool approached a steady constant value [9]. The agreement between stability predictions and theoretical results are very good.

In Figs. 6 and 7, plots of the arising vibration frequencies are accompanied to the stability charts. The theoretical chatter frequencies of Hopf type $\left(f_{H}\right)$ and of period doubling type $\left(f_{P D}\right)$ are denoted by continuous
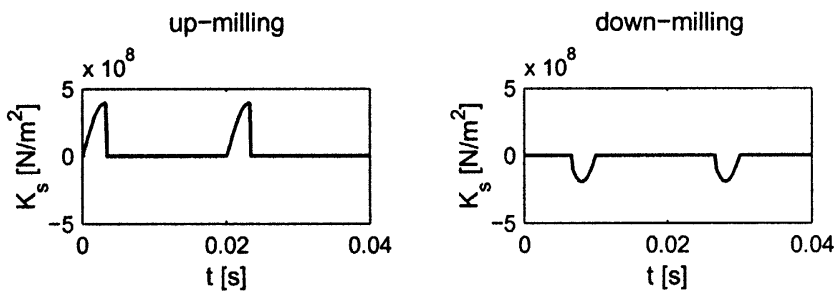

Fig. 5. Theoretical specific cutting force variation for $a / D=0.237$. 

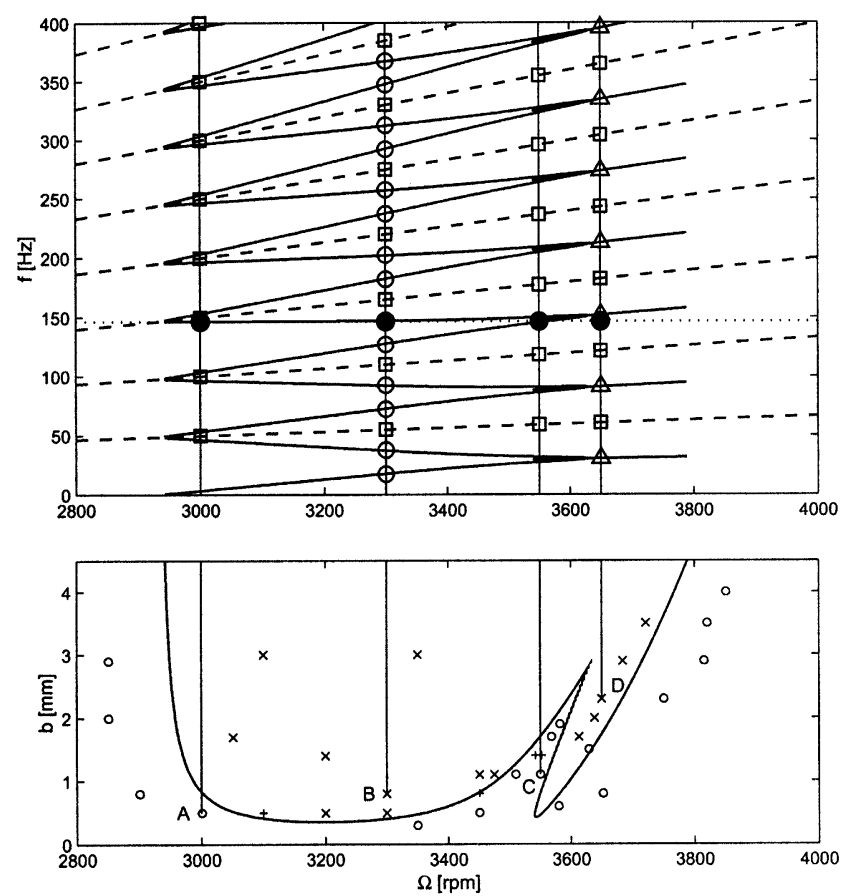

Fig. 6. Up-milling analytical stability boundaries vs. experimental results $(\bigcirc-$ stable cutting, $\times$ - chatter, + - border $)$ and analytical vibration frequencies $\left(\bigcirc-f_{H}, \triangle-f_{P D}, \square-f_{T P E}, \bullet-f_{d}\right)$.
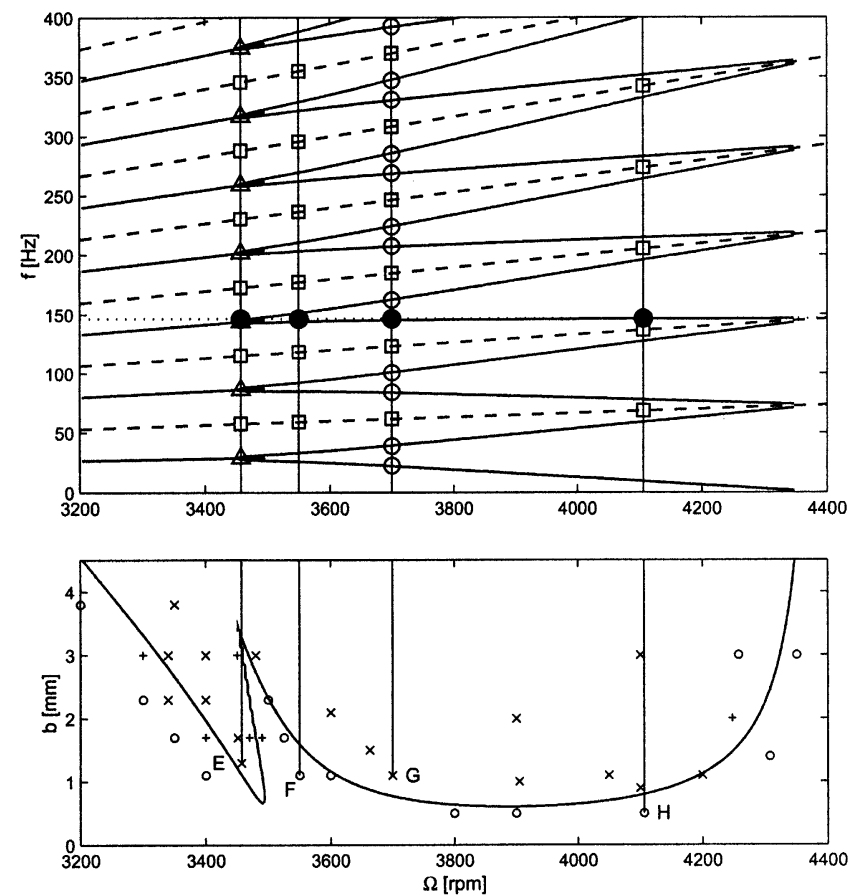

Fig. 7. Down-milling analytical stability boundaries vs. experimental results $(\bigcirc-$ stable cutting, $\times$ - chatter, + - border) and analytical vibration frequencies $\left(\bigcirc-f_{H}, \triangle-f_{P D}, \square-f_{T P E}, \bullet-f_{d}\right)$. lines, the tooth pass excitation frequencies $\left(f_{T P E}\right)$ are denoted by dashed lines and a dotted line denotes the damped natural frequency of the flexure at $f_{d}=$ $f_{n} \sqrt{1-\zeta^{2}} \approx 146.5 \mathrm{~Hz}$. For both up- and down-milling cases, four points are emphasized: A $(\Omega=3000 \mathrm{rpm}$, $b=0.5 \mathrm{~mm}), \quad$ B $\quad(\Omega=3300 \mathrm{rpm}, \quad b=0.8 \mathrm{~mm})$, $C(\Omega=3550 \mathrm{rpm}, b=1.1 \mathrm{~mm}), D(\Omega=3650 \mathrm{rpm}$, $b=2.3 \mathrm{~mm})$ for up-milling, and $\mathrm{E}(\Omega=3450 \mathrm{rpm}$, $b=1.3 \mathrm{~mm}), \quad \mathrm{F} \quad(\Omega=3550 \mathrm{rpm}, \quad b=1.1 \mathrm{~mm}), \quad \mathrm{G}$ $(\Omega=3700 \mathrm{rpm}, \quad b=3700 \mathrm{rpm}, \quad b=1.1 \mathrm{~mm}), \quad \mathrm{H}$ $(\Omega=4100 \mathrm{rpm}, b=0.5 \mathrm{~mm})$ for down-milling. The frequencies related to these points are denoted by various symbols: $\bigcirc$ for $f_{H}, \triangle$ for $f_{P D}, \square f_{T P E}$ and $\bullet$ for $f_{d}$.

Points A, C, F and $\mathrm{H}$ are related to stable, chatter-free machining. For these cases, only $f_{T P E^{-}}$and $f_{d}$-frequencies are expected.

Points B and G are related to unstable behaviour predicted in the theoretical model by complex eigenvalues with a magnitude greater than one. These correspond to Hopf bifurcations. In this case, two additional $f_{H}$-frequencies are predicted in the neighbourhood of each $f_{T P E}$-frequencies.

Points D and E are related to period doubling, when the dominant eigenvalue is negative and real with a magnitude greater than one. In this case, the chatter becomes a subharmonic of order 2 , and $f_{P D}$-frequencies show up in the middle of two $f_{T P E}$-frequencies.

The experimental power spectra of the data sets corresponding to these 8 points are shown in Figs. 8 and 9. The symbols mentioned above help to identify all the various frequency sets. The comparison of the theoretical
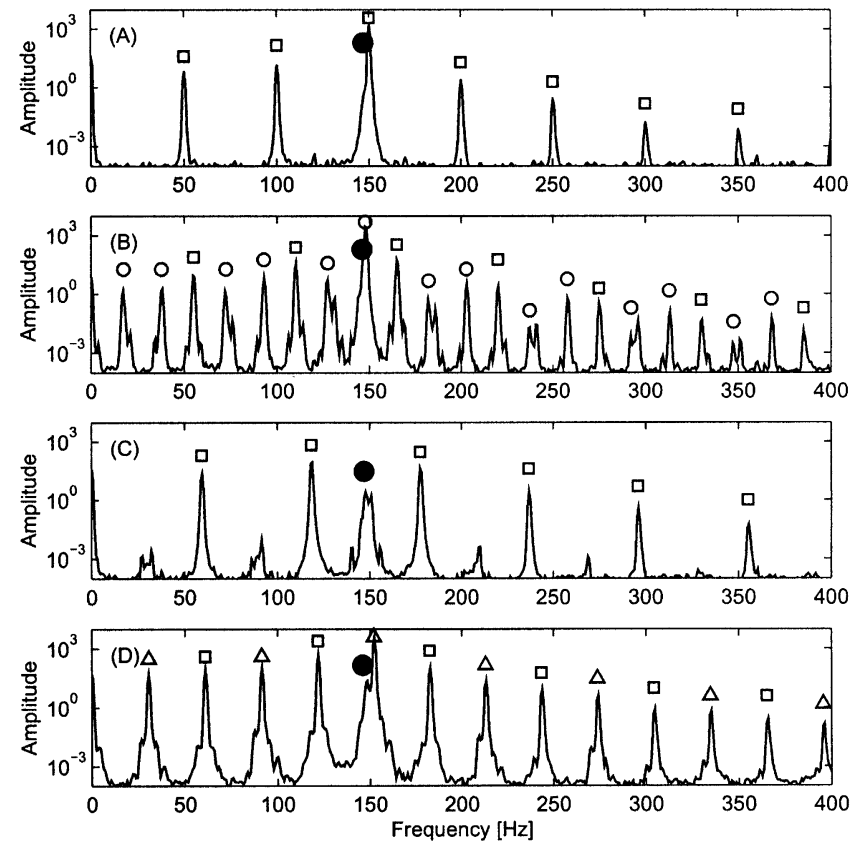

Fig. 8. Power spectra for parameter points A, B, C and D of Fig. 6 $\left(\bigcirc-f_{H}, \triangle \longrightarrow f_{P D}, \square-f_{T P E}, \bullet-f_{d}\right)$. 

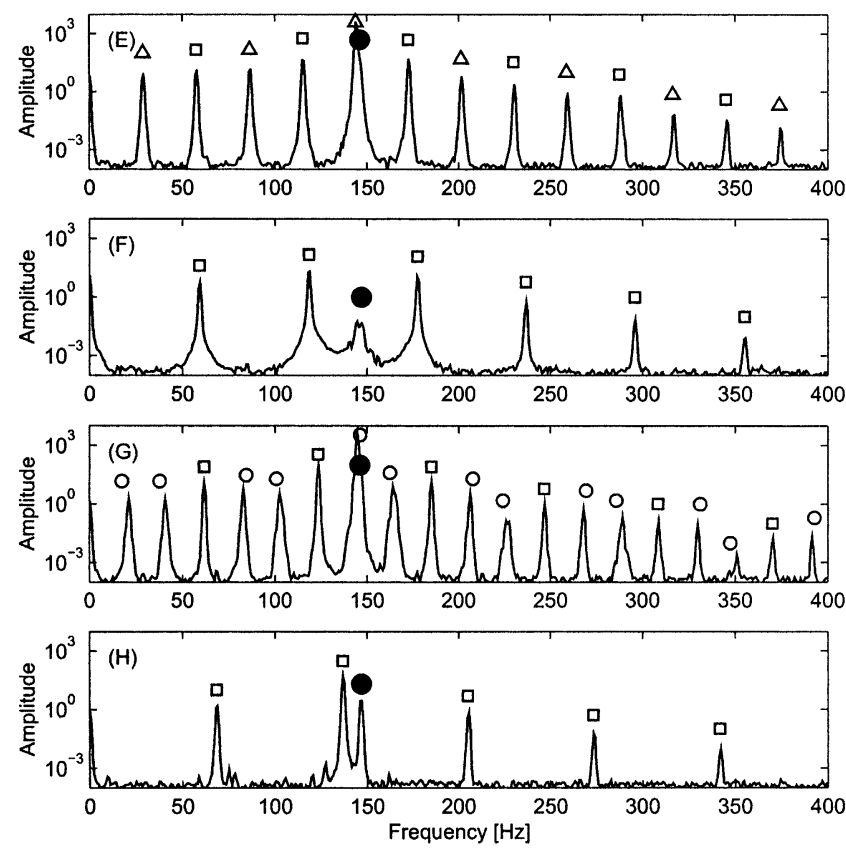

Fig. 9. Power spectra for parameter points E, F, G and H of Fig. 7 $\left(\bigcirc-f_{H}, \triangle \longrightarrow f_{P D}, \square-f_{T P E}, \bullet-f_{d}\right)$.
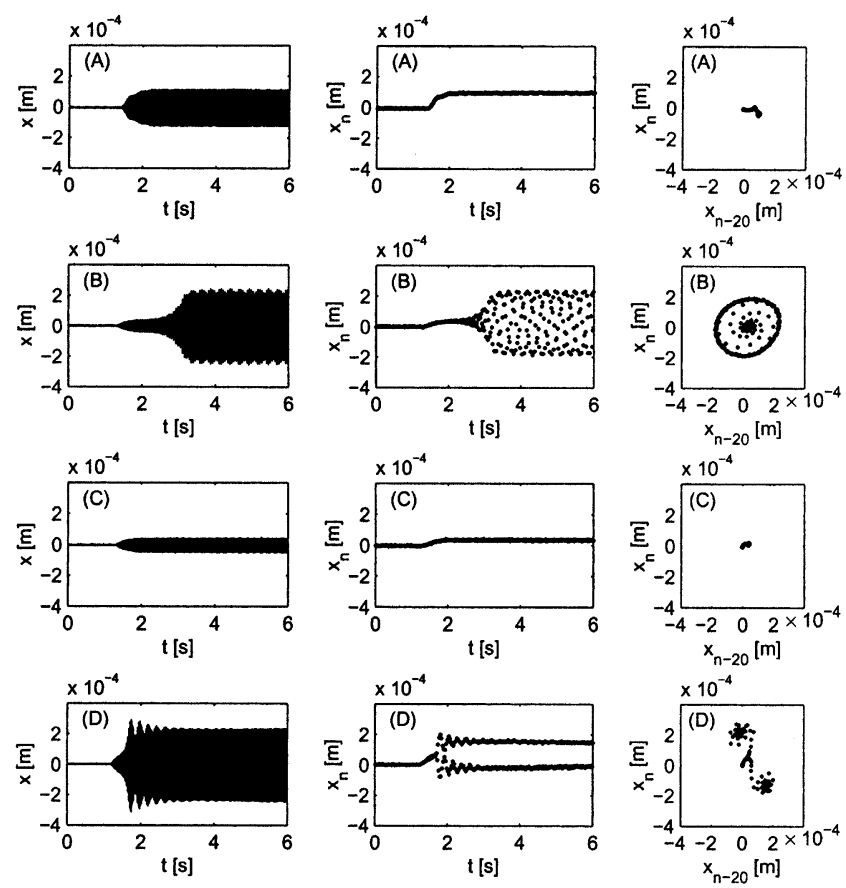

Fig. 10. Continuous time histories, 1/rev sampled signals, and Poincaré sections for points A, B, C and D of Fig. 6.

frequencies in Figs. 6 and 7 and the experimental power spectra in Figs. 8 and 9 shows good agreement.

Raw measurements and once per cutter revolution data samples are shown in Fig. 10 for up-milling and in Fig. 11 for down-milling. The 1/rev displacement samples are used to construct experimental Poincaré sections as discussed by Virgin [15]. Data sets A, C, F and
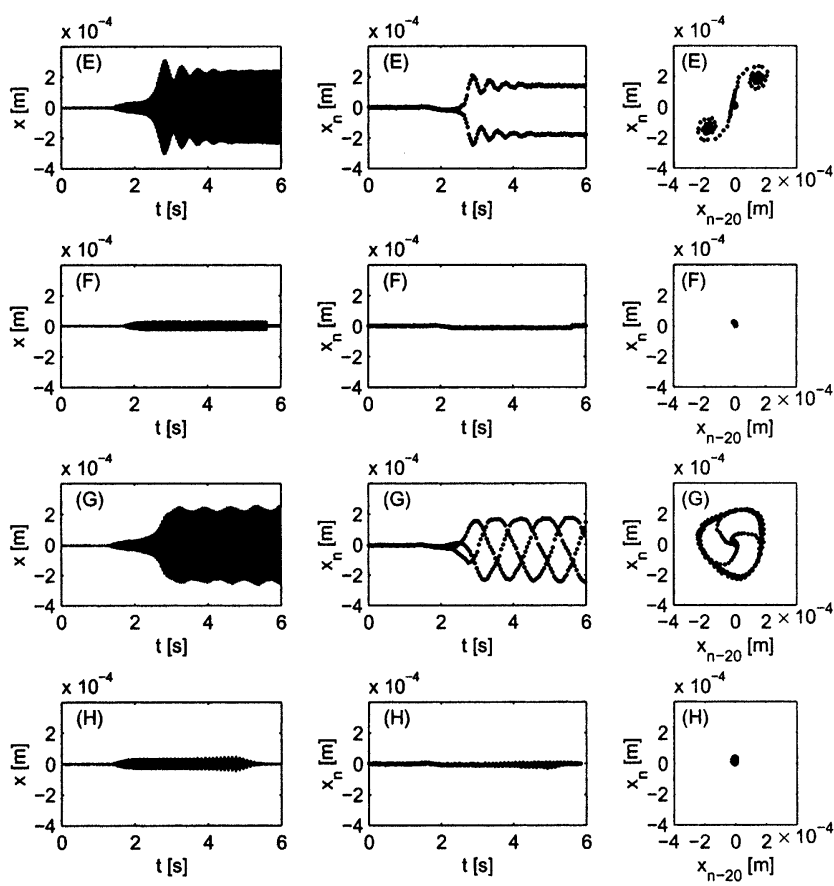

Fig. 11. Continuous time histories, $1 /$ rev sampled signals, and Poincaré sections for points $\mathrm{E}, \mathrm{F}, \mathrm{G}$ and $\mathrm{H}$ of Fig. 7.

$\mathrm{H}$ of Fig. 10 and Fig. 11 are clear examples of stable behaviour. Although the vibration amplitude of each of these data sets differ in magnitude, each $1 /$ rev plot shows that the cutting tooth returns to the same position each time it hits the surface.

For Hopf type instabilities, the chatter vibrations are generally unsynchronised with tooth passage. This results a quasiperiodic motion as shown by data set $\mathrm{B}$ in Fig. 10. For data set $\mathrm{G}$ in Fig. 11, a kind of period triple phenomenon can be observed. This is a special case of quasiperiodic motions, when the two governing frequencies are multiple of each other. In this case, the smallest tooth pass excitation frequency $\left(f_{T P E}\right)_{\text {min }} \approx 62 \mathrm{~Hz}$ is about the triple of the smallest chatter frequency $\left(f_{H}\right)_{\min } \approx 20.5 \mathrm{~Hz}$, as it is shown by the power spectrum of data set $G$ in Fig. 9. This means that the critical characteristic multipliers are the unit roots $\mu_{1,2}=\exp ( \pm \mathrm{i} 2 \pi / 3)$, and the cutting tool returns to the same position after three revolution of the tool. For the data set $\mathrm{B}$, the governing frequencies are $\left(f_{T P E}\right)_{\text {min }} \approx 55 \mathrm{~Hz}$ and $\left(f_{H}\right)_{\text {min }} \approx 17 \mathrm{~Hz}$, and they are not a simple multiple of each other.

The phenomenon of period doubling is shown in data set $\mathrm{D}$ in Fig. 10 and data set $\mathrm{E}$ in Fig. 11. In this case, there exists a characteristic multiplier $\mu_{1}=\exp (\mathrm{i} \pi)=$

-1 , and the cutting tool returns to the same position after two revolution of the tool. 


\section{Summary and conclusions}

In this paper, a SDOF model for low radical immersion up-milling and down-milling is validated experimentally. In the companion paper [14], two essentially different methods, the FEAT and the SD methods, are used to transform the governing equations into approximate discrete maps. The eigenvalues of these maps (the so-called characteristic multipliers) are used to determine the stability of the system. Eigenvalues with magnitude greater than one indicate instability. The FEAT and the SD methods converge to the same result.

Experimental evidence confirms the analytical stability predictions for both the case of up-milling and down-milling. The experiments also show that different stability behaviour may occur for up-milling and downmilling at the same parameter combinations (depth of cut, and spindle speed). In particular, the relocation of the quasiperiodic chatter phenomenon (Hopf lobe) for the case of down-milling was confirmed. The experimental observation of this change in the sequence of the Hopf lobes and the flip lobes for the cases of up- and down-milling has a practical relevance for the design of high-speed technological parameters.

The carefully identified frequency branches of quasiperiodic chatter, period doubling chatter and those of the tooth pass excitation, gave a rigorous validation of the mechanical model and its analytical and numerical investigation. Time signals were determined experimentally for stable and unstable cutting operations, period doubling and quasiperiodic motions were identified by spectral analysis, too. A kind of period triple behaviour was also detected, as a special case of quasiperiodic chatter.

The constructed test rig allows exploration of system stability behaviour in a SDOF interrupted cutting process. It is not intended to replicate a particular industrial cutting process, but instead to focus on the stability mechanisms. The relatively low natural frequency of the system allows the investigation of cutting dynamics typically seen at much higher spindle speed.

Since an actual high-speed milling process usually involves at least two degrees of freedom, future analytical and experimental work will focus on expanding the methods presented to more realistic models with multiple degrees of freedom.

\section{Acknowledgements}

This research was supported by the US National Science Foundation Grants DMI-9900108 (GOALI) and CMS-9625161 (CAREER) and the U.S. National Defense Science and Engineering Graduate Fellowship, the Hungarian National Science Foundation under grant no. OTKA T030762.

\section{References}

[1] E. Kondo, H. Ota, T. Kawai, Regenerative chatter vibrations of turning workpiece, Transactions of the Japan Society 58 (1992) 1251-1265.

[2] J.E. Halley, A.M. Helvey, K.S. Smith, W.R. Winfough, The impact of high-speed machining on the design and fabrication of aircraft components, in: Proceedings of 1999 ASME Design and Technical Conferences, Las Vegas, Nevada (1999) paper no. DETC99/VIB-8057 (CD-ROM).

[3] G. Yucesan, Y. Altintas, Improved modeling of cutting force coefficients in peripheral milling, International Journal of Machine Tools and Manufacture 34 (4) (1994) 473-487.

[4] J. E. Halley, Stability of low radial immersion milling, MSc Thesis, Washington University, St. Louis, Missouri, 1999.

[5] D.J. Inman, Engineering Vibration, 2nd ed., Prentice-Hall, Upper Saddle River, New Jersey, 2001.

[6] J.-N. Juang, Applied System Identification, Prentice-Hall, Englewood Cliffs, New Jersey, 1994.

[7] J. Gradišek, E. Govekar, I. Grabec, Time series analysis in metal cutting: chatter versus chatter-free cutting, Mechanical Systems and Processing 12 (6) (1998) 839-854.

[8] J. Gradišek, E. Govekar, I. Grabec, Using coarse-grained entropy rate to detect chatter in cutting, Journal of Sound and Vibration 214 (5) (1998) 941-952.

[9] T.L. Schmitz, M.A. Davies, K. Medicus, J. Snyder, Improving high-speed machining material removal rates by rapid dynamic analysis, Annals of the CIRP 50 (1) (2001) 263-268.

[10] A. Sitz, U. Schwarz, J. Kurths, D. Maus, M. Wiese, G. Warnecke, Signatures of acoustic emission signals generated during high speed cutting, in: Proceedings of the ASME 2001 Design Engineering Technical Conferences, Pittsburgh, Pennsylvania (2001) paper no. DETC2001/VIB-21618 (CD-ROM).

[11] J. Gradišek, E. Govekar, I. Grabec, Analysis of data from periodically forced stochastic processes, Physics Letters A 294 (3-4) (2002) 234-238.

[12] G. Stépán, Retarded Dynamical Systems, Longman, Harlow, 1989.

[13] T. Insperger, G. Stépán, P.V. Bayly, B.P. Mann, Multiple chatter frequencies in milling processes, Journal of Sound and Vibration (2002) in press.

[14] T. Insperger, B. P. Mann, G. Stépán, P. V. Bayly, Stability of upmilling and down-milling, Part 1: Alternative analytical methods, International Journal of Machine Tools and Manufacture (2002) submitted.

[15] L.N. Virgin, Introduction to Experimental Nonlinear Dynamics, Cambridge University Press, Cambridge, 2000. 